\title{
NOTA CIENTIFICA
}

\section{Presencia de microplásticos en cuatro playas arenosas de Perú}

\author{
Microplastic in four sandy beaches from Peruvian coast
}

\section{Sara Purca* y Aida Henostroza}

Área Funcional de Investigaciones Marino Costeras (AFIMC), Dirección General de Investigaciones en Acuicultura (DGIA), IMARPE. Esquina Gral. Gamarra y Valle S/N-Chucuito Callao.

*Autor para correspondencia

Email Sara Purca: spurca@imarpe.gob.pe

Email Aida Henostroza: ahenostroza@imarpe.gob.pe

\begin{abstract}
Resumen
Se presenta el número y peso por metro cuadrado de fragmentos de microplásticos presentes en cuatro playas arenosas de la costa peruana. Las muestras fueron colectadas entre junio de 2014 y mayo 2015. Fragmentos de plástico duro mayores a $1 \mathrm{~mm}$ fueron encontrados en más del $80 \%$ de las muestras de las cuatro playas. La playa Costa Azul ( 12 $\left.{ }^{\circ} \mathrm{S}\right)$ presentó 522 fragmentos por metro cuadrado (items $\left./ \mathrm{m}^{2}\right)$ de microplásticos, de los cuales, 463.33 items $/ \mathrm{m}^{2}$ y $2.6 \mathrm{~g} / \mathrm{m}^{2}$ fueron plásticos duros. La playa Albúfera de Medio Mundo ( $\left.11^{\circ} \mathrm{S}\right)$ presentó el menor número y peso de microplásticos (4.67 items $/ \mathrm{m}^{2}$ y $\left.0.50 \mathrm{~g} / \mathrm{m}^{2}\right)$. Una muestra aleatoria de la playa Costa Azul fue analizada por el espectroscopio FT-IR. Se encontró 5 fragmentos con poliuretano (PE), dos fragmentos con polipropileno (PP) y un fragmento con estireno (EPS). Pocos estudios mencionan al plástico duro como la mayor fracción de los microplásticos, se sugiere nuevas fuentes de intrusión y vías de microbasura en los hábitas, los cuales podrían estar afectando desde la base de trama trófica marina en el Perú.
\end{abstract}

Palabras clave: Basura marina; microbasura; playas arenosas; Perú.

\section{Abstract}

Results of measurement of the microplastic number and weigh from four sandy beaches along Peruvian coast are shown. Microplastic samples were collected during June 2014 to May 2015. Hard plastic fragment > 1 $\mathrm{mm}$ was found at over $80 \%$ of the four sampling locations. The Costa Azul beach $\left(\sim 12^{\circ} \mathrm{S}\right)$ presented the 522 items per $\mathrm{m}^{2}$ of microplastic, in which the 463.33 items per $\mathrm{m}^{2}$ and weight $2.6 \mathrm{~g}$ per $\mathrm{m}^{2}$ were hard plastic. The Medio Mundo $\left(\sim 11^{\circ} \mathrm{S}\right)$ beach has the lowest microplastic in number and weight $\left(4.67\right.$ items per $\mathrm{m}^{2}$ and 0.50 $\mathrm{g}$ per $\mathrm{m}^{2}$ ). The random microplastic sample from Costal Azul beach was confirmed by FT-IR spectroscopy, we found five fragments with polyurethane (PE), two pieces with polypropylene (PP) and one piece of Styrofoam (EPS). Few studies mention hard plastic with a main component of microplastic, and we suggest new insights into the sources and pathway of microdebris into habitats.

Keywords: Marine debris; microdebris; microplastic; sandy beaches; Peru.

\section{Citación:}

Purca S. \& A. Henostroza. 2017. Presencia de microplásticos en cuatro playas arenosas de Perú. Revista peruana de biología 24(1): 101 - 106 (Abril 2017). doi: http://dx.doi.org/10.15381/rpb.v24i1.12724
Información sobre los autores:

SP Y AH: realizaron el diseño experimental; realizaron los muestreos analizaron los datos; redactaron, revisaron y aprobaron el manuscrito. Los autores no incurren en conflictos de intereses.

$\begin{array}{ll}\text { Presentado: } & 29 / 11 / 2016 \\ \text { Aceptado: } & 03 / 02 / 2017\end{array}$

$\begin{array}{ll}\text { Aceptado: } & 03 / 02 / 2017 \\ \text { Publicado online: } 20 / 04 / 2017\end{array}$ 


\section{Introducción}

En todo el mundo uno de los impactos humanos que recientemente está incrementándose y representa un peligro para la vida marina es la contaminación por plásticos en los oceános (Derraik 2002). Se ha reportado fibras sintéticas microscópicas en el sedimento, en la zona de alta marea, así como en la columna de agua y estuarios del Reino Unido; la mayor cantidad de fibras se presentó en las zonas de alta marea donde se identificaron nueve tipos de polímeros: acrílico, alcalinos, poli (etileno-propileno), poliamida, poliéster, nylon, polietileno, polimetilacrílico y polivinil alcohol (Thompson et al. 2004). Estos polímeros son ampliamente usados en la industria de la ropa, pesca y el empaque.

En Sudamérica una de las revisiones más actualizadas sobre los estudios de los desechos plásticos ha sido realizada en Brasil (Ivar do Soul \& Costa 2007). Aunque, estos estudios presentan altos niveles de detalle (identificación, distribución espacial y temporal de patrones ecológicos y consecuencias de conservación en mamíferos), aún permanecen en desarrollo los diagnósticos tempranos y los reportes básicos sobre los problemas de ingestión de plásticos por la biota marina (Ivar do Sul \& Costa 2007). En las últimas décadas, serias amenazas se han reconocido con relación a la ingestión de microplásticos por peces, aves, tortugas y mamíferos marinos, (Andrady 2011, Derraik 2002, Tomas et al. 2002, Jantz et al. 2013). Un reciente estudió sobre el efecto de las partículas de plástico en la dieta de larvas de peces, sugirió que el impacto ecológico de las partículas de poliestireno en la dieta de las larvas sería negativo provocando una disminución nutricional (Lönnstedt \& Eklöv 2016).

Se ha propuesto una clasificación de las dimensiones concernientes a los tamaños de los microplásticos iguales o menores a $5 \mathrm{~mm}$ hasta nanomicras. Los microplásticos han sido clasificados en primarios y secundarios (Cole et al. 2013). Los primarios están comprendidos en tamańos microscópicos, usualmente son usados como limpiadores y exfoliadores faciales. Los microplásticos secundarios han sido clasificados en tamaños que se pueden percibir a simple vista entre 5 a $1 \mathrm{~mm}$.

Los microplásticos son comúnmente estudiados en relación a 1) muestras plancton, 2) sedimentos y barro marino, 3) ingestión en vertebrados y invertebrados, e interacciones químicas contaminantes (Ivar do Sul and Costa 2014). Los efectos de los microplásticos en la biota marina han sido determinados en estudios con la detección en orden de milímetros de fragmentos plásticos y pellets tanto en el Atlántico (Ivar do Sul and Costa 2014) como en el Pacífico (Hidalgo-Ruz et al. 2012). Además, los microplásticos pueden absorber y acumular tóxicos y compuestos persistentes en el mar (PBT, polibutileno tereftalato) (Gouin et al. 2011), el cual incluye compuestos orgánicos persistentes (POPs, Persistent Organic Pollutants) y metales (Rios et al. 2007, Ashton et al. 2010), los cuales afectarían los organismos marinos.

De acuerdo al trabajo de Rochman y colaboradores (2013), la toxicidad en el mar producida por la adhesión de partículas de metal y otras sustancias tóxicas a las partículas de plástico como los bifenilos policlorados (PCB) e hidrocarburos aromáticos policíclicos (PHA), así como otros tipos de contaminantes químicos representan un coctel de contaminación, donde el polímero que acumula menos partículas tóxicas es el Tereftalato de polietileno (PET), de amplio uso.
En Lima, entre los años 2009 al 2013 la generación de residuos sólidos por habitante reportado por sus municipios se ha incrementado (MINAM 2016). Pero no se reportan volúmenes de plásticos encontrados en estos residuos. La contaminación en el litoral peruano ha sido consecuencia principalmente del aumento de los usos de la zona costera. Las principales ciudades se han desarrollado en la costa peruana, con un aumento significativo del uso de plásticos en actividades agrícolas y riego tecnificado, actividades pesqueras y acuícolas, transporte marítimo, minería, explotación y transporte de hidrocarburos, desechos de la construcción, desechos urbanos y turismo, los cuales representan una fuente de generación de basura marina que podría afectar la trama trófica del mar peruano. En relación a la generación potencial de basura marina, representada en su mayoría por desechos plásticos, a inicios de la década de los 90 , se ha reportado que el Perú produce 1'695425 toneladas por ańo a lo largo de la costa (CPPS 2007).

En el presente estudio, nosotros describimos la presencia de los fragmentos de microplásticos en número y peso por metro cuadrado en cuatro playas arenosas de la costa peruana, además de un análisis de los polímeros encontrados en 10 (diez) fragmentos de microplásticos.

\section{Material y métodos}

Se evaluaron cuatro playas arenosas a lo largo de la costa peruana (Fig. 1), playa Vesique $\left(9.29^{\circ} \mathrm{S}, 78.17 \mathrm{oW}\right)$, playa Albúfera de Medio Mundo (11.37oS, $\left.77.57^{\circ} \mathrm{W}\right)$, playa Costa Azul -Ventanilla $\left(12.08^{\circ} \mathrm{S}, 77.08^{\circ} \mathrm{W}\right)$; y playa El Chaco $\left(13.72^{\circ} \mathrm{S}, 76.3^{\circ} \mathrm{W}\right)$ cuyas características morfológicas se explican en la Tabla 1.

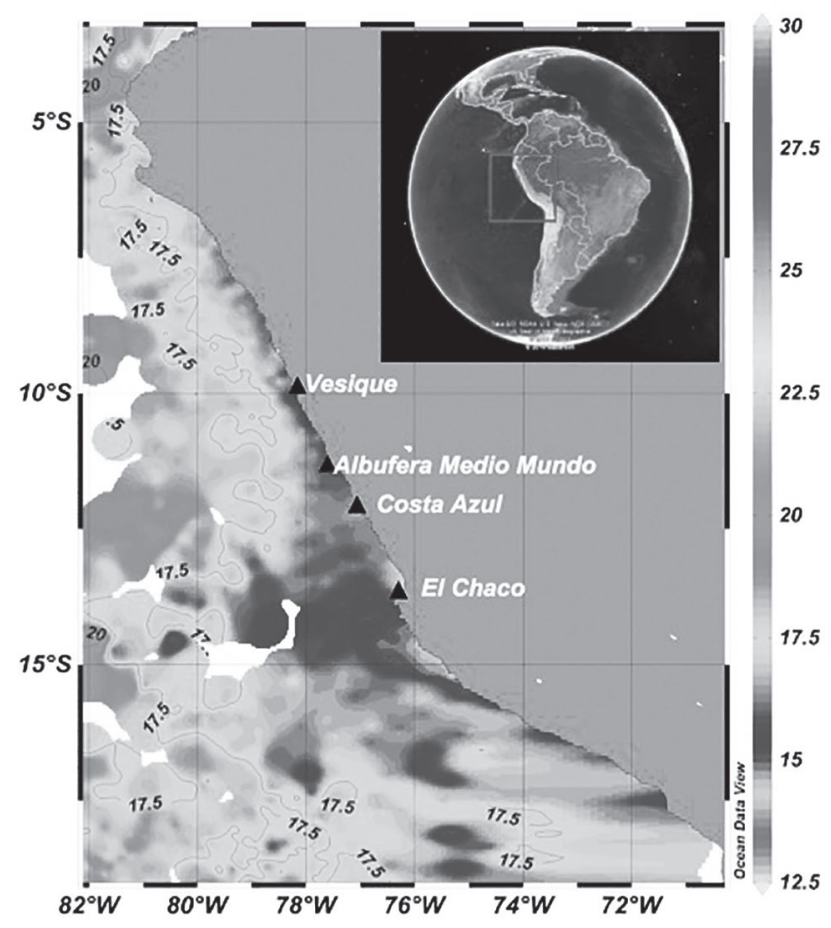

Figura 1. Localización de las estaciones de muestreo a lo largo de la costa peruana (triángulos). Los colores en el mapa representan la temperatura superficial del mar promedio entre los meses de Julio a setiembre para el periodo 2000 a 2012 de la base de datos mundial oceanográfica WOD2009. 
Tabla 1. Características morfológicas de las cuatro playas arenosas de la costa peruana.

\begin{tabular}{lcccccc}
\hline Playas & Latitud $\left({ }^{\circ} \mathbf{S}\right)$ & Longitud $\left({ }^{\circ} \mathbf{W}\right)$ & viento predominante & Curvatura de la playa & Tipo de sustrato & Perfil horizontal \\
\hline $\begin{array}{l}\text { Playa Vesique } \\
\text { Playa Albúfera de }\end{array}$ & 9.29 & 78.17 & Sur (DHN, 1995) & concava & arena fina & $0^{\circ}$ de inclinación \\
$(\mathrm{DHN}, 1999)$
\end{tabular}

Las muestras fueron colectadas entre junio 2014 y mayo 2015 con el protocolo del Instituto Tecnológico y Ciencia del Océano de Corea (KIOST, 2016) desarrollado en el curso de entrenamiento ambiental marino para la cooperación Asia Pacífico (AMETEC, 2014). Se eligieron playas arenosas que presentaban más de 100 metros de largo. Cada playa se dividió en dos transectos con tres cuadrantes, para delimitar los cuadrantes se utilizó un marco de 0.5 por $0.5 \mathrm{~m}$. La primera fila de cuadrantes se ubicó sobre la línea de acarreo (parte alta de la zona supralitoral).
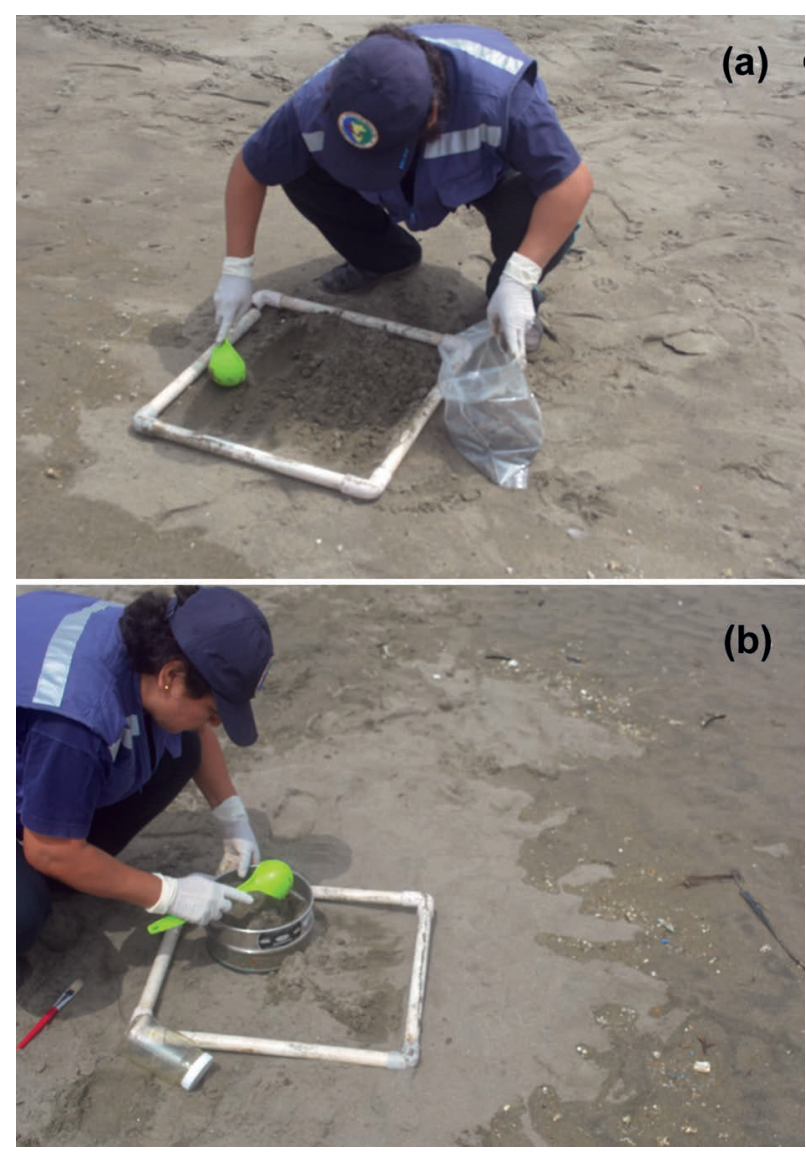

Figura 2. Toma de muestra de microplásticos: a. Recolección de la capa superficial en un marco de $0.5 \mathrm{~m}$ por $0.5 \mathrm{~m}$ en una bolsa de plástico con un cucharon, b. Tamizado de la capa superficial para la obtención de partículas mayores a un milímetro.
Las muestras fueron colectadas de la capa superficial con un cucharón y tamizadas usando un tamiz de $1 \mathrm{~mm}$. Los fragmentos atrapados en el tamiz se guardaron en una bolsa con cierre. Las muestras mayores a $2.5 \mathrm{~mm}$ fueron removidas; desechos orgánicos, como son los fragmentos de ramas y semillas son removidos. Para evaluar la presencia de fragmentos menores a un milímetro, la arena tamizada de los seis cuadrantes se juntaron en una bandeja, se homogenizó y luego se colectó tres cuartos o la mitad de un frasco de $500 \mathrm{ml}$ para su posterior análisis (Fig. 2).

En el laboratorio los fragmentos mayores a $1 \mathrm{~mm}$ se contaron sin necesidad de un microscopio, luego se pesaron en una balanza analítica. Los microplásticos fueron clasificados en nueve categorías de plásticos y se guardaron en placas Petri. La clasificación de microplásticos fue: 1. plástico duro, 2. otras espumas, 3. fibra, 4. estireno, 5. pellets blanco, 6. pellet negro, 7. Pellet transperente, 8. fragmento de film, 9. otros polímeros (Fig. 3).

Todos los valores obtenidos de tamaño y peso se normalizan a un metro cuadrado para el análisis estadístico descriptivo.

Se seleccionaron 10 piezas de plástico duro para su análisis con el espectrómetro infrarojo por transformada de Fourier (siglas en inglés FT-IR) marca Bruker, Tensor 27, este análisis fue realizado en el laboratorio del KIOST.

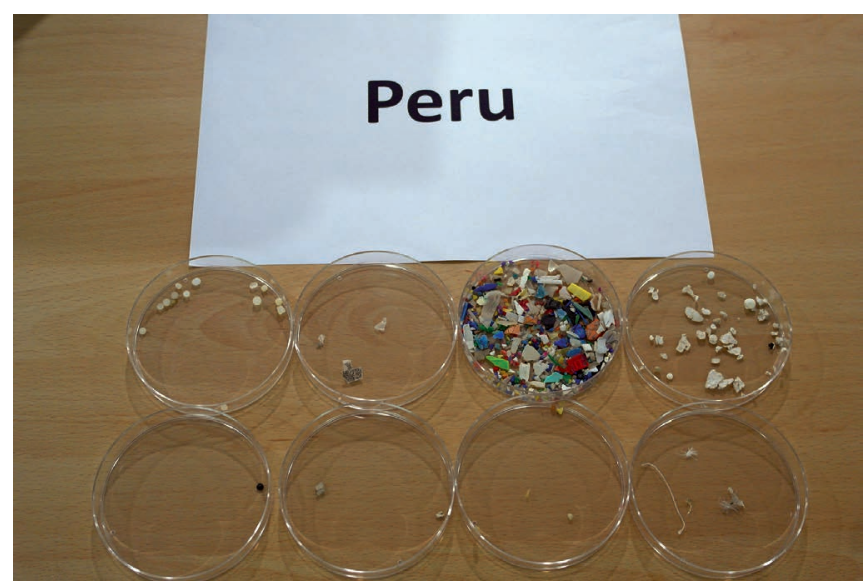

Figura 3. Clasificación según tipos de plásticos en placas petri. Foto de las muestras obtenidas de las muestras llevadas de la Playa costa Azul al curso AMETEC 2014, KIOST, campus sur, Corea. 
Finalmente, en el laboratorio se añadió agua de mar a cada frasco hasta completar la capacidad máxima del mismo, luego en una campana extractora se filtró cada frasco con una bomba de vacío utilizando un filtro de fibra de vidrio, cuyo tamaño de poro fue de $1 \mu \mathrm{m}$. Cada filtro se guardó en una placa Petri para su posterior análisis.

\section{Resultados}

Fragmentos de plástico duro mayores a $1 \mathrm{~mm}$ fueron encontrados en las cuatro playas arenosas y representaron más del $80 \%$ de cada muestra. La Playa Vesique presentó 40 items* $\mathrm{m}^{-2}$ $\left(0.95 \mathrm{~g}^{*} \mathrm{~m}^{-2}\right)$, la playa Albúfera de Medio Mundo 4.67 items $^{*} \mathrm{~m}^{-2}$ $\left(0.50 \mathrm{~g}^{*} \mathrm{~m}^{-2}\right)$, la playa Costa Azul, Ventanilla 463.33 items per $\mathrm{m}^{2}\left(2.6 \mathrm{~g}^{*} \mathrm{~m}^{-2}\right)$ y la Playa El Chaco presentó 11.33 items* $\mathrm{m}^{-2}$ $\left(0.86 \mathrm{~g}^{*} \mathrm{~m}^{-2}\right)$ (Fig. 4)

La composición según los tipos de plásticos de la playa Costa Azul fueron: $89 \%$ de plástico duro, $7 \%$ de estireno y $2 \%$ pellets negro, $1 \%$ de otras espumas, $1 \%$ polímero, $1 \%$ fibra, and $1 \%$ de láminas transparentes (Tabla 2).

La composición espectral determinada por el FT-IR de 10 fragmentos de microplásticos de la playa Costa Azul fueron: cinco fragmentos de poliuretano, un fragmento fue espuma de estireno, y otra pieza de estireno. La densidad del polímero fue: 3.3 poliuretano (PE) y 1.33 de polipropileno (PP).

\section{Discusión}

Los fragmentos de plásticos duros encontrados en nuestra investigación pertenecen a la clasificación de plásticos secundarios menores a cinco milímetros, esta clasificación toma en cuenta las características físicas y morfológicas (tamaño, forma y color) y nos permiten determinar su distribución en el ambiente y su biodisponibilidad a los organismos (Imhof et al. 2012, Cole et al. 2013). Pero también, factores como, la evaluación de las conexiones hidrodinámicas (turbulencia, densidad, circulación, etc.), actividades socio económicas urbanas e industriales, así como factores meteorológicos pueden determinar la distribución y la procedencia de los microplásticos de alta densidad de las zonas litorales urbanas (v.g. Eriksen et al. 2013), así nuestros resultados podrían ser utilizados en este mismo propósito.

En zonas oceánicas, modelos de transporte desarrollados en la región este-centro del giro del Pacífico Subtropical Sur $(20-$ $\left.40^{\circ} \mathrm{S}, 120-80^{\circ} \mathrm{W}\right)$, muestran las trayectorias de los fragmentos de basura marina asociada a las corrientes superficiales, los cuales una vez que llegan a esta área quedan atrapados (Martinez et al. 2009).

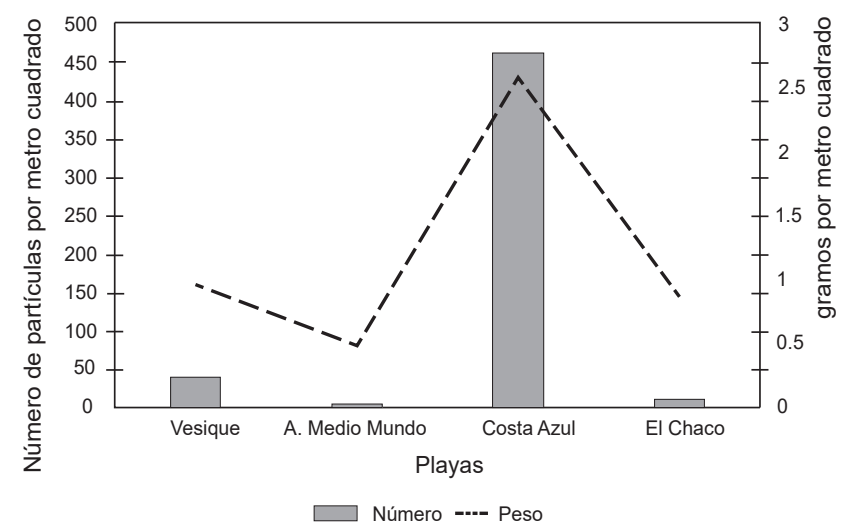

Figura 4. Número y peso de las muestras de microplásticos duros de cuatro playas de la costa de Perú: playa Vesique $\left(9.29^{\circ} \mathrm{S}, 78.17^{\circ} \mathrm{W}\right)$, playa Albúfera de Medio Mundo (11.37 $\mathrm{S}$, $77.57^{\circ} \mathrm{W}$ ), playa Costa Azul -Ventanilla (12.08 $\left.{ }^{\circ} \mathrm{S}, 77.08^{\circ} \mathrm{W}\right)$; y playa El Chaco $\left(13.72^{\circ} \mathrm{S}, 76.3^{\circ} \mathrm{W}\right)$.

En promedio, en las zonas costeras usualmente se encuentra más de 20 items por metro cuadrado (Thiel et al. 2003) hasta cientos de miles de partículas (Rocha-Santos \& Duarte 2015). En nuestro estudio, una de las posibles fuentes serían los residuos urbanos de las bahías del Callao y Miraflores para los fragmentos de plásticos duros encontrados en la playa Costa Azul, mientras que los fragmentos encontrados en las playas de Vesique, Albúfera de Medio Mundo y el Chaco podrían originarse de las actividades de pesca, turismo y recreación. En el borde costero de Lima y Callao, el sistema de desagüe y alcantarillado para una población de 8 millones 890 mil 792 y 1 millón 13 mil 935 habitantes en Lima y Callao, respectivamente (INEI 2015), representan las mayores descargas de colectores hacia el mar, ambos desembocan en las bahías del Callao y Miraflores, donde el sistema de corrientes costero retiene los residuos de basura marina en un pequeńo giro con sentido antihorario frente a la playa Costa Azul, Ventanilla (Correa et al. 2008).

En relación a los tamaños de microplásticos se han realizado revisiones extensas donde concluyen que hay una predominancia de fragmentos plásticos en superficie del mar y sedimento con un rango de tamańos de $500 \mu \mathrm{m}$ a $5 \mathrm{~mm}$, en relación a lo encontrado en columna de agua (Hidalgo-Ruz et al. 2012). En este trabajo se obtuvo fragmentos de plásticos duros en un rango de $1 \mathrm{~mm}$ a $2.5 \mathrm{~mm}$, nuestros resultados muestran 522 items de microplásticos por metro cuadrado en la playa Costa

Tabla 2. Tipos de plásticos encontrados en la muestra de playa Costa Azul, Ventanilla.

\begin{tabular}{|c|c|c|c|c|c|}
\hline Tipos de plástico & & $\mathbf{N}^{\circ}$ de partículas & Partículas /metro cuadrado & Peso (g) & Peso (gramos/metro cuadrado) \\
\hline \multirow{3}{*}{ Pellet } & Transparente & 0 & & & \\
\hline & Blanco & 14 & & 0.263 & \\
\hline & Color & 1 & & & \\
\hline Plástico duro & & 695 & 463.33 & 3.903 & 2.602 \\
\hline Capa & & 4 & & 0.023 & \\
\hline Fibra & & 8 & & 0.01 & \\
\hline Estireno (tecnopor) & & 54 & & 0.07 & \\
\hline Otras espumas & & 5 & & 0.006 & \\
\hline Otros polímeros & & 2 & & 0.013 & \\
\hline TOTAL & & & 522 & & 2.6833 \\
\hline
\end{tabular}


Tabla 3. Muestreo estratificado de microplásticos realizado en la playa Costa Azul, Ventanilla en mayo 2016. Los números romanos representan los transectos.

\begin{tabular}{ccccccccc}
\hline $\begin{array}{c}\text { Zonas Playa Costa Azul- } \\
\text { Ventanilla }\end{array}$ & I & II & III & IV & V & VI & Número total & Peso total (g) \\
\hline & & & & & & & & \\
Línea de alta marea & 4 & 10 & 3 & 1 & 1 & 1 & 20 & 3570 \\
Línea marea & 0 & 2 & 3 & 3 & 2 & 2 & 12 & 5330 \\
Línea de baja marea & 2 & 4 & 1 & 4 & 1 & 1 & 13 & 0.385 \\
\hline
\end{tabular}

Azul, además se encontró un gradiente de partículas de mayor a menor partículas desde la zona supralitoral (alta marea) hasta la zona infralitoral (baja marea) (Tabla 3), esta característica también fue observado en el trabajos a lo largo de la costa de Chile (Hidalgo-Ruz et al. 2012).

Una revisión sobre los tipos de microplásticos encontrados en diferentes ambientes acuáticos mostró en mayor proporción fragmentos de fibras de redes de pesca que otros tipos de plástico en la zona supralitoral de las costas de Reino Unido (Thompson et al. 2004), pocos trabajos mencionan que encuentran plásticos duros en un rango de 1 a $5 \mathrm{~mm}$, como en este trabajo realizado en cuatro playas arenosas de Perú, por ejemplo veinticuatro investigaciones de cuarenta y cuatro trabajos sobre microplásticos mencionaron que encontraron en mayor proporción pellets en sedimentos y playas arenosas (Hidalgo-Ruz et al. 2012). Nuestro trabajo aporta mayor información sobre el impacto de las grandes ciudades sobre la zona costera, donde el impacto de la microbasura marina se está incrementado a nivel mundial (Jambeck et al. 2015).

Por otro lado, en relación a la proporción de polímeros que conforman los plásticos duros se encontró en este estudio se reporta 3.3 poliuretano $(\mathrm{PE})$ partículas $/ \mathrm{m}^{2}$, and 1.33 polipropileno (PP) partículas $/ \mathrm{m}^{2}$. Otros trabajos muestran que la composición química de los microplásticos se han encontrado polietileno (PE), polipropileno (PP) and polystireno (PS) (Hidalgo-Ruz et al. 2012). Los organismos marinos, en especial los grupos más vulnerables como organismos del zooplancton y larvas de peces, serían los grupos más afectados por la presencia de microplásticos en la columna de agua, aunque este trabajo está orientado a la búsqueda de microplásticos en la zona supralitoral, la presencia de microplásticos en nuestras playas representan una alterta sobre el posible impacto de estos fragmentos en la alimentación de especies marinas en la base de la trama trófica (Ivar do Sul \& Costa 2014).

En las aguas costeras del Pacífico Sudeste entre los 18 y $50^{\circ} \mathrm{S}$, se ha observado que más del $80 \%$ de los fragmentos marinos flotantes son plásticos, cuyo origen provienen de centros portuarios (Thiel et al. 2003), usualmente actividades de mitigación como la reutilización, el reciclaje y la reducción de uso de plásticos en las actividades industriales y urbanas han motivado campañas de educación sobre la problemática de la basura marina en todos los niveles de educación, sin embargo pocos países están dispuestos a eliminar el uso de plásticos en utensilios domésticos, bolsas de supermercado y residuos. El presente trabajo muestra que nuevos tipos de plásticos estarían conformando los microplásticos y estas serían nuevas vías de contaminación en los hábitats marinos que deben ser estudiadas.

\section{Agradecimientos}

Este trabajo ha sido realizado en el área funcional de investigaciones marino costeras (AFIMC), de la Dirección General de Investigaciones Acuícolas (DGIA) del Instituto del Mar del Perú (IMARPE) y se agradece a todo el personal de investigación, técnico y administrativo de IMARPE, en especial a la Blga. Sulma Carrasco por proponer la participación de SP en el curso sobre microplásticos AMETEC 2014 en KIOST- Geoje, Corea del Sur.

\section{Literatura citada}

AMETEC (Curso de entrenamiento ambiental marino para la cooperación Asia Pacífico). 2014. How to use AMETEC protocol on beach debris survey?. Korea Marine Litter Institute. Yong Chang Jang, Jongmyoung Lee, Sunwook Hong, Jong Su Lee (Eds.). AMETEC Workshop, June 30 - July 7, 2014. South Sea Research Institute (KIOST), Geoje- Korea

Andrady A.L. 2011. Microplastics in the marine environment. Marine Pollution Bulletin 62: 1596-1605. http://dx.doi. org/10.1016/j.marpolbul.2011.05.030

Ashton K.L., L. Holmes \& A. Turner. 2010. Association of metals with plastic production pellets in the marine environment. Marine Pollution Bulletin 60: 2050-2055. http://dx.doi. org/10.1016/j.marpolbul.2010.07.014

Cole M., P. Lindeque, E. Fileman et al. 2013. Microplastic ingestion by zooplankton. Environmental Science \& Technology 47 (12): 6646-6655. http://dx.doi.org/10.1021/es400663f

Correa D., J. Tam., J Pasapera, M. Saavedra, A. Ingunza. 2008. Modelado de la circulación marina y descargas hipotéticas en la bahñia del Callao, Perú. Instituto del Mar de Perú, Informe, 35 (3):181-192. http://biblioimarpe.imarpe.gob.pe:8080/ handle/123456789/1967 acceso 29/11/2016

CPPS. 2007. Basura Marina en el Pacífico Sudeste: una revisión del problema. Comisión Permanente del Pacífico Sur. Guayaquil, Ecuador. 31 pp.

Derraik J.G.B. 2002. The pollution of the marine environment by plastic debris: a review. Marine Pollution Bulletin 44: 842852. http://doi.org/10.1016/S0025-326X(02)00220-5

Dirección de Hidrografía y Navegación de la Marina. 1995. Derrotero de la Costa del Perú. Volumen I. 169pp.

Dirección de Hidrografía y Navegación de la Marina. 1999. Derrotero de la Costa del Perú. Volumen I. 274pp.

Eriksen M., S. Mason, S. Wilson, C. Box, A. Zellers, W. Edwards, H. Farley \& S. Amato. 2013. Microplastic pollution in the surface waters of the Laurentian Great Lakes. Marine Pollution Bulletin 77 (1-2): 177-82. http://doi.org/10.1016/S0025326X(02)00220-510.1016/j.marpolbul.2013.10.007.

Gouin T., N. Roche, R. Lohmann, G. Hodges. 2011. A thermodynamic approach for assessing the environmental exposure of chemicals absorbed to microplastic. Environmental Science \& Technology 45(4): 1466-1472. http://dx.doi. org/10.1021/es1032025

Imhof H.K., J. Schmid, R. Niessner, N. Ivleva, C. Laforsch. 2012. A novel, highly efficient method for the separation and quantification of plastic particles in sediments of aquatic environments. Limnology and oceanography: methods 10, 2012, 524-537. http://dx.doi.org/10.4319/lom.2012.10.524

INEI 2015. Estado de la Población Peruana 2015. https://www.inei. gob.pe/media/MenuRecursivo/publicaciones_digitales/Est/ 
Lib1251/Libro.pdf , Acceso 25/11/2016.

Ivar do Sul J.A. \& M.F. Costa. 2007. Marine debris review for Latin America and the Wider Caribbean Region: from the 1970 until now and where do we go from here?. Marine Pollution Bulletin 54: 1087-1104. http://dx.doi.org/10.1016/j. marpolbul.2007.05.004

Ivar do Sul J.A. \& M.F. Costa. 2014. The present and future of microplastic pollution in the marine environment. Environmental Pollution 185: 352-364. http://dx.doi.org/10.1016/j. envpol.2013.10.036

KIOST. 2016. South Sea Research Institute. Acceso 29/11/2016 en: www.kiost.ac.kr/cyber_en/sub.html?menu_cd=0301

Hidalgo-Ruz V., L. Gutow, R.C. Thompson et al. 2012. Microplastics in the Marine Environment: A Review of the Methods Used for Identification and Quantification. Environmental Science \& Technology 46(6): 3060-75. http://dx.doi. org/10.1021/es2031505

Jantz L.A., C.L. Morishige, G.L. Bruland et al. 2013. Ingestion of plastic marine debris by longnose lancetfish (Alepisaurus ferox). Marine Pollution Bulletin 69: 97-104 doi: http:// dx.doi.org/10.1016/j.marpolbul.2013.01.019

Jambeck J.R., R. Geyer, C. Wilcox, T.R. Siegler, M. Perryman, A. Andrady, R. Narayan, K. Lavander Law. 2015. Plastic waste imputs from land into the ocean. Science. 347(6223), 768$771 \mathrm{http}: / / \mathrm{dx}$.doi.org/10.1126/science. 1260352

Lönnstedt O.O. \& P. Eklöv. 2016. Environmentally relevant concentrations of microplastic particles influence larval fish ecology. Science 352:1213-1216. http://dx.doi.org/10.1126/science. aad 8828

Martinez E., K. Maamaatuaiahutapu, V. Taillandier. 2009. Floating marine debris surface drift: Convergence and accumulation toward the South Pacific subtropical gyre. Marine Pollution Bulletin 58: 1347-1355. http://dx.doi.org/10.1016/j.marpolbul.2009.04.022

MINAM (Ministerio del Ambiente). 2016. Sistema Nacional de Información Ambiental. <http://sinia.minam.gob.pe/indicador/1007> Acceso 27/10/16.

Rios L.M., C. Moore \& P.R. Jones .2007. Persistent organic pollutants carried by synthetic polymers in the ocean environment. Marine Pollution Bulletin 54: 1230-1237 http://dx.doi. org/10.1016/j.marpolbul.2007.03.022

Rochman C.M., E. Hoh, B.T. Hentschel et a. 2013. Long-Term Field Measurement of Sorption of Organic Contaminants to Five Types of Plastic Pellets: Implications for Plastic Marine Debris. Environmental. Science \& Technology 47(3): 16461654. http://dx.doi.org/10.1021/es303700s

Rocha-Santos T. \& A.C. Duarte. 2015. A critical overview of the analytical approaches to the occurrence, the fate and the behavior of microplastics in the environment. Trends in Analytical Chemistry 65, 47-53. http://dx.doi. org/10.1016/j.trac.2014.10.011

Thiel M., I. Hinojosa, N. Vásquez, E. Macaya. 2003. Floating marine debris in coastal waters of the SE-Pacific (Chile). Marine Pollution Bulletin. 46, 224-231. http://doi.org/10.1016/ S0025-326X(02)00365-X

Thompson R.C., Y. Olsen, R.P. Mitchell et al. 2004. Lost at Sea: Where Is All the Plastic?. Science 304: 838. http://doi.org/10.1126/ science. 1094559

Tomás J., R. Guitart, R. Mateo et al. 2002. Marine debris ingestion in loggerhead sea turtles, Caretta caretta from the Western Mediterranean. Marine Pollution Bulletin 44: 211-216. http://doi.org/10.1016/S0025-326X(01)00236-3 